TRANSACTIONS OF THE

AMERICAN MATHEMATICAL SOCIETY

Volume 359, Number 5, May 2007, Pages 2383-2394

S 0002-9947(06)04078-5

Article electronically published on December 15, 2006

\title{
INDEPENDENCE OF HECKE ZETA FUNCTIONS OF FINITE ORDER OVER NORMAL FIELDS
}

\author{
MACIEJ RADZIEJEWSKI
}

\begin{abstract}
We study oscillations of the remainder term corresponding to the counting functions of the sets of elements with unique factorization length in semigroups of algebraic numbers such as the semigroup of algebraic integers or totally positive algebraic integers in a given normal field $K$. The results imply existence of oscillations when the exponent of the class group of the semigroup in question is sufficiently large depending on the field's degree. In particular, when $K$ is a quadratic field or a normal cubic field oscillations exist whenever the class group is not isomorphic to $C_{2}^{a} \oplus C_{3}^{b} \oplus C_{4}^{c}$ for nonnegative integers $a, b, c$. The main part of this study is concerned with the problem of multiplicative independence of Hecke zeta functions. We also show that there are infinitely many fields whose Dedekind zeta function has infinitely many nontrivial multiple zeros.
\end{abstract}

\section{IntRodUCtion AND STATEMENT OF RESULTS}

Let $K$ be a normal algebraic number field, $\mathcal{O}_{K}$ its ring of algebraic integers, $\mathcal{I}\left(\mathcal{O}_{K}\right)$ the semigroup of nonzero ideals, and $H^{*}(K)$ the class group of $K$ in the narrow sense. We denote by $S_{\Gamma}$ the semigroup of nonzero ideals of $\mathcal{O}_{K}$ whose classes belong to a subgroup $\Gamma$ of $H^{*}(K)$. The semigroup $S_{\Gamma}$ is a special case of the generalized Hilbert semigroup defined by F. Halter-Koch [4, Beispiel 4]; cf. also [3. In particular, for appropriate choices of $\Gamma$, we recover the reduced multiplicative semigroup of $\mathcal{O}_{K}$ (the case studied most extensively) or the reduced semigroup of totally positive algebraic integers in $K$, with multiplication.

$S_{\Gamma}$ is a Krull monoid (cf. [4). We denote its class group by $\mathrm{Cl}\left(S_{\Gamma}\right)$ and its class number by $h$. We identify characters of $\mathrm{Cl}\left(S_{\Gamma}\right) \cong H^{*}(K) / \Gamma$ with the corresponding characters of $H^{*}(K)$ and $\mathcal{I}\left(\mathcal{O}_{K}\right)$. We recall that the Galois group $\operatorname{Gal}(K / \mathbf{Q})$ acts on $H^{*}(K)$ and hence on the characters group $\left.\widehat{H^{*}(K}\right)$. Let $\operatorname{Gal}\left(S_{\Gamma}\right)$ denote the set of automorphisms $\sigma \in \operatorname{Gal}(K / \mathbf{Q})$ such that $\sigma(\Gamma)=\Gamma$. Clearly $\operatorname{Gal}\left(S_{\Gamma}\right)$ acts on $\mathrm{Cl}\left(S_{\Gamma}\right)$ and on the characters group $\widehat{\mathrm{Cl}\left(S_{\Gamma}\right)}<\widehat{H^{*}(K)}$. Suppose further that the orbits of $\chi \in \widehat{\mathrm{Cl}\left(S_{\Gamma}\right)}$ in the actions of $\operatorname{Gal}(K / \mathbf{Q})$ and $\operatorname{Gal}\left(S_{\Gamma}\right)$ are the same. In the most important cases named above we have in fact $\operatorname{Gal}\left(S_{\Gamma}\right)=\operatorname{Gal}(K / \mathbf{Q})$, because

Received by the editors November 6, 2004 and, in revised form, April 16, 2005.

2000 Mathematics Subject Classification. Primary 11N64.

Key words and phrases. Functional independence, Selberg class, oscillations, error terms, Mellin transforms, Hilbert semigroup, factorizations of distinct lengths.

This work was supported by the Foundation for Polish Science and by the Polish Research Committee (KBN grant No. 1P03A00826).

(C)2006 American Mathematical Society Reverts to public domain 28 years from publication 
$\Gamma$ is invariant under the action of $\operatorname{Gal}(K / \mathbf{Q})$. Let

$$
\zeta(s, \chi)=\sum_{\mathfrak{a} \in \mathcal{I}\left(\mathcal{O}_{K}\right)} \frac{\chi(\mathfrak{a})}{\mathrm{N}(\mathfrak{a})^{s}}, \quad \operatorname{Re} s>1,
$$

denote the Hecke zeta function corresponding to $\left.\chi \in \widehat{H^{*}(K}\right)$ and $\zeta_{K}(s)$ the Dedekind zeta function. Let $\mathcal{S}$ denote the Selberg class (see, e.g., 7] or [6]). For any complex function $F(s)$ regular in a certain half-plane $\operatorname{Re} s>\sigma_{0}$ and non-vanishing in a halfplane $\operatorname{Re} s \geq \sigma_{1}>\sigma_{0}$, such that $\arg F(\sigma)$ is close to 0 when $\sigma$ is real and large, we choose the branch of $\log F(s)$ such that $\operatorname{Im} \log F(\sigma)$ is close to 0 when $\sigma$ tends to $+\infty$ and extend it in the unique way to the half-plane $\sigma>\sigma_{0}$ with cuts from the edge of the half-plane to the zeros of $F(s)$. Following this convention $\log s$ stands for the principal branch of the logarithm and $\log \mathcal{S}$ denotes the set of logarithms of functions from $\mathcal{S}$ (cf. [6). The multiplicity of a zero of a complex function $F(s)$ at $s=\varrho, \varrho \in \mathbf{C}$, is written as $m(\varrho, F)$. For a set $A \subseteq S_{\Gamma}$ the zeta function associated to $A$ is

$$
\zeta(s, A)=\sum_{\mathfrak{a} \in A} \frac{1}{\mathrm{~N}(\mathfrak{a})^{s}}, \quad \operatorname{Re} s>1 .
$$

We use the notation $\langle U\rangle$ for the subgroup of $\mathrm{Cl}\left(S_{\Gamma}\right)$ generated by $U$, and $\langle\chi \mid U\rangle$ for the scalar product of $\chi \in \widehat{\mathrm{Cl}\left(S_{\Gamma}\right)}$ and the characteristic function of $U \subseteq \mathrm{Cl}\left(S_{\Gamma}\right)$ :

$$
\langle\chi \mid U\rangle=\frac{1}{h} \sum_{X \in U} \chi(X)
$$

$C_{n}$ is a cyclic group of order $n, \varphi$ is Euler's function, and $|U|$ the number of elements of a set $U$. Let $G_{1}$ be the set of elements with a unique factorization length in $S_{\Gamma}$, i.e. elements $\mathfrak{a} \in S_{\Gamma}$ such that every factorization of $\mathfrak{a}$ into irreducibles in $S_{\Gamma}$ has the same length. Let $G_{1}(x)$ denote the counting function of $G_{1}$, i.e. the number of elements $\mathfrak{a} \in G_{1}$ with $\mathrm{N}(\mathfrak{a}) \leq x$. It is well known (cf. [1]) that $G_{1} \neq S_{\Gamma}$ if and only if $h \geq 3$.

We study irregularities in the distribution of elements with a unique factorization length in $S_{\Gamma}$. Our results imply the existence of oscillations of the counting function $G_{1}(x)$ around its main term (in a sense made precise further on) for $K$ and $S_{\Gamma}$ such that the exponent of $\mathrm{Cl}\left(S_{\Gamma}\right)$ is sufficiently large, depending on the degree of $K$. In particular, oscillations exist when $K$ is a quadratic field or a normal cubic field, and $\mathrm{Cl}\left(S_{\Gamma}\right)$ is not isomorphic to $C_{2}^{a} \oplus C_{3}^{b} \oplus C_{4}^{c}$ for nonnegative integers $a, b, c$.

In [10] we studied irregularities in the distribution of elements of various sets of algebraic numbers with prescribed factorization properties, including $G_{1}$. The oscillatory behaviour of the function $G_{1}(x)$ could then only be shown with extra assumptions, either combinatorial (cf. also 12]) or analytic (related to multiplicities of zeros of Hecke zeta functions), that we conjectured to be always satisfied. Here we do not assume such conjectures, but restrict ourselves to the case of normal fields.

Our results are based on the study of multiplicative independence (i.e. linear independence of logarithms) of Hecke zeta functions associated to characters of $H^{*}(K)$. We use it in the investigation of singularities induced by products of complex powers of Hecke zeta functions. Multiplicative independence is also an assumption of the Functional Independece Theorem of J. Kaczorowski and A. Perelli [6], although it is not essential for the application of this theorem (cf. [10, 6]). It may 
also be interesting in its own right. We show that in the case of a normal field distinct Hecke zeta functions are always multiplicatively independent.

Theorem 1. If $K$ is a normal field, then:

(i) If $\psi_{1}, \ldots, \psi_{k} \in \widehat{H^{*}(K)}$ and the functions $\zeta\left(s, \psi_{1}\right), \ldots, \zeta\left(s, \psi_{k}\right)$ are distinct, then $\log \zeta\left(s, \psi_{1}\right), \ldots, \log \zeta\left(s, \psi_{k}\right)$ are linearly independent over $\mathbf{Q}$.

(ii) For $\chi, \psi \in \widehat{H^{*}(K)}$ the functions $\zeta(s, \chi)$ and $\zeta(s, \psi)$ are equal if and only if $\chi=\psi \circ \sigma$ for some $\sigma \in \operatorname{Gal}(K / \mathbf{Q})$.

Corollary 1. If $K$ is a quadratic number field, $\chi, \psi \in \widehat{H^{*}(K)}$, then the functions $\zeta(s, \chi)$ and $\zeta(s, \psi)$ are equal if and only if $\chi=\psi$ or $\chi=\bar{\psi}$.

Corollary 2. There are infinitely many nonisomorphic algebraic number fields whose Dedekind zeta functions have infinitely many nontrivial multiple zeros.

It should be noted that the multiple zeros in the last corollary are directly related to $\zeta_{K}(s)$ being divisible by a higher power of another $L$-function. If $\zeta_{K}(s)$ is a product of distinct primitive functions in the Selberg class we expect that all but finitely many of its nontrivial zeros are simple, according to the "simple zeros" and "distinct zeros" conjectures (cf. 7] for these and other conjectures concerning the Selberg class).

The description of elements with a unique factorization length is based on the theory of half-factorial sets. For a subset $U$ of an additively written finite abelian group $G$, a block over $U$ is a formal product $\prod_{g \in U} g^{\alpha_{g}}$ with $\alpha_{g} \in \mathbf{N} \cup\{0\}, g \in U$, such that the sum $\sum_{g \in U} \alpha_{g} g$ equals zero; cf. [8] and [9, Chapter 9]. The set $\mathcal{B}(U)$ of such blocks with multiplication is a Krull monoid. The set $U$ is called half-factorial if each irreducible element $\prod_{g \in U} g^{\alpha_{g}}$ of $\mathcal{B}(U)$ satisfies

$$
\sum_{g \in U} \frac{\alpha_{g}}{\operatorname{ord} g}=1
$$

cf. e.g. 14, 16, 2]. Let $\mu(G)$ be the maximum cardinality of a half-factorial subset of $G$.

The function $\zeta\left(s, G_{1}\right)$ can be shown to be regular around $\left[\frac{1}{2}, 1\right]$ except for the real points to the left of $\frac{1}{2}$; cf. Section 2 , Let $\mathcal{C}$ be a contour starting at $\frac{1}{2}-\delta$, for a small $\delta>0$, going closely around $\left[\frac{1}{2}, 1\right]$, counterclockwise, and back to $\frac{1}{2}-\delta$. We call

$$
M(x)=\frac{1}{2 \pi i} \int_{\mathcal{C}} \zeta\left(s, G_{1}\right) \frac{x^{s}}{s} d s, \quad x \geq 1,
$$

the main term of $G_{1}(x)$, similar to [5] and [6, theorem 3].

We say that a real, piecewise continuous function $f(x)$ is subject to oscillations of lower logarithmic frequency $\gamma$ and size $x^{\theta-\varepsilon}$ (for $\gamma \in(0,+\infty], \theta \in \mathbf{R}$ ) if there exists an increasing sequence of positive real numbers $\left(x_{n}\right)_{n=1}^{\infty}, \lim _{n \rightarrow \infty} x_{n}=\infty$, such that:

(1) We have $f\left(x_{n}\right) \neq 0$ for each $n$ and the signs of $f\left(x_{n}\right)$ alternate.

(2) If $V(Y)$ denotes the number of terms of $\left(x_{n}\right)$ not exceeding $Y$, then

$$
\liminf _{Y \rightarrow \infty} \frac{V(Y)}{\log Y}=\gamma
$$

(3) If $\varepsilon>0$, then for any $Y$ sufficiently large the segment, $\left[Y^{1-\varepsilon}, Y\right]$ contains at least one element of $\left(x_{n}\right)$. 
(4) We have

$$
\liminf _{n \rightarrow \infty} \frac{\left|f\left(x_{n}\right)\right|}{x_{n}^{\theta-\varepsilon}}=+\infty
$$

for every $\varepsilon>0$.

Theorem 2. Suppose $h \geq 3$. Suppose there exists a half-factorial $U \subset \mathrm{Cl}\left(S_{\Gamma}\right)$ with $|U|=\mu\left(\mathrm{Cl}\left(S_{\Gamma}\right)\right)$ and $X, Y \in \mathrm{Cl}\left(S_{\Gamma}\right)$ such that $\langle X\rangle=\langle Y\rangle$, and the orbits of $X$ and $Y$ in the action of $\operatorname{Gal}\left(S_{\Gamma}\right)$ have a different number of elements in $U$. Then the error term $G_{1}(x)-M(x)$ is subject to oscillations of positive lower logarithmic frequency and size $x^{\frac{1}{2}-\varepsilon}$.

Corollary 3. If $\mathrm{Cl}\left(S_{\Gamma}\right)$ has a half-factorial subset $U$ with $|U|=\mu\left(\mathrm{Cl}\left(S_{\Gamma}\right)\right)$ containing an element $X \in U$ such that $\varphi($ ord $X)>\left|\operatorname{Gal}\left(S_{\Gamma}\right)\right|$, then the error term $G_{1}(x)-M(x)$ is subject to oscillations of positive lower logarithmic frequency and size $x^{\frac{1}{2}-\varepsilon}$.

Corollary 4. If $K$ is a quadratic field or a normal cubic field and there exists a half-factorial set $U \subset \mathrm{Cl}\left(S_{\Gamma}\right)$ with $|U|=\mu\left(\mathrm{Cl}\left(S_{\Gamma}\right)\right)$ containing an element $X \in U$ of order other than $1,2,3,4,6$, then the error term $G_{1}(x)-M(x)$ is subject to oscillations of positive lower logarithmic frequency and size $x^{\frac{1}{2}-\varepsilon}$.

In a joint paper with Wolgang A. Schmid [12 we show, using combinatorial methods, that if $\mathrm{Cl}\left(S_{\Gamma}\right)$ has a half-factorial subset $U$ with $|U|=\mu\left(\mathrm{Cl}\left(S_{\Gamma}\right)\right)$ not generating $\mathrm{Cl}\left(S_{\Gamma}\right)$, then $\mathrm{Cl}\left(S_{\Gamma}\right)$ satisfies a combinatorial condition necessary for the application of Theorem 4 (Section 2, cf. also [10]), and $G_{1}(x)-M(x)$ is again subject to oscillations. On the other hand, if $U$ is a generating subset of $\mathrm{Cl}\left(S_{\Gamma}\right)$ and $\varphi(\operatorname{ord} X) \leq\left|\operatorname{Gal}\left(S_{\Gamma}\right)\right|$ for all $X \in U$, then we note that there are only finitely many $m \in \mathbf{N}$ such that $\varphi(m) \leq\left|\operatorname{Gal}\left(S_{\Gamma}\right)\right|$. Hence the exponent of $\mathrm{Cl}\left(S_{\Gamma}\right)$ divides the least common multiple of all such $m$. Therefore Corollary 3 implies the existence of oscillations of $G_{1}(x)-M(x)$ provided that the exponent of $\mathrm{Cl}\left(S_{\Gamma}\right)$ is sufficiently large, depending on the degree of $K$. Corollary 4 implies the existence of oscillations in the case of quadratic and normal cubic fields when $\mathrm{Cl}\left(S_{\Gamma}\right)$ is not isomorphic to $C_{2}^{a} \oplus C_{3}^{b} \oplus C_{4}^{c}$ for nonnegative integers $a, b, c$.

The author wishes to thank Professor Jerzy Kaczorowski for his help during the preparation of this paper. Constructive remarks of the anonymous referee are gratefully acknowledged.

\section{Preliminaries}

Let $\Omega_{X}(\mathfrak{a})$ denote the number of prime ideal divisors of $\mathfrak{a} \in S_{\Gamma}$ belonging to the class $X \in \mathrm{Cl}\left(S_{\Gamma}\right)$, counted according to their multiplicities. For $U \subseteq \mathrm{Cl}\left(S_{\Gamma}\right)$ and $A: \operatorname{Cl}\left(S_{\Gamma}\right) \backslash U \rightarrow \mathbf{N} \cup\{0\}$ we put

$$
N_{U, A}=\left\{\mathfrak{a} \in S_{\Gamma}: \Omega_{X}(\mathfrak{a})=A(X), X \in \mathrm{Cl}\left(S_{\Gamma}\right) \backslash U\right\}
$$

The set $G_{1}$ is a union of a finite number of sets $N_{U, A}$ with half-factorial $U$; cf. [13, 14, 15. This allows us to describe the analytic properties of the Mellin transform of $G_{1}(x)$ using the functions $\zeta\left(s, N_{U, A}\right)$; cf. also [5, 10]. 
Lemma 1. We have

$$
\begin{aligned}
\int_{0}^{\infty} G_{1}(x) x^{-s-1} d x & =\frac{1}{s} \zeta\left(s, G_{1}\right) \\
& =\frac{1}{s} \sum_{i=1}^{m} \alpha_{i} \zeta\left(s, N_{U_{i}, A_{i}}\right), \quad \operatorname{Re} s>1,
\end{aligned}
$$

for some $U_{i} \subseteq \mathrm{Cl}\left(S_{\Gamma}\right)$ half-factorial, $A_{i}: \mathrm{Cl}\left(S_{\Gamma}\right) \backslash U_{i} \rightarrow \mathbf{N} \cup\{0\}, \max _{i}\left|U_{i}\right|=$ $\mu\left(\mathrm{Cl}\left(S_{\Gamma}\right)\right)$, and integers $\alpha_{1}, \ldots, \alpha_{m}$ such that $\alpha_{i}>0$ whenever $\left|U_{i}\right|=\mu\left(\mathrm{Cl}\left(S_{\Gamma}\right)\right)$.

Lemma 2 ([10]). For every $U \subset \mathrm{Cl}\left(S_{\Gamma}\right), A: \operatorname{Cl}\left(S_{\Gamma}\right) \backslash U \rightarrow \mathbf{N} \cup\{0\}$, we have

$$
\begin{aligned}
\zeta\left(s, N_{U, A}\right)= & \left(\frac{1}{h} \sum_{\chi} \chi(Y) \prod_{\psi} \zeta(s, \psi)\langle\chi \bar{\psi} \mid U\rangle \cdot \prod_{X \in U} F_{X, \chi(X)}(s)\right) \\
& \cdot \prod_{X \notin U} P_{X, A(X)}\left(\log \zeta\left(s, \chi_{0}\right), \ldots, \log \zeta\left(s, \chi_{h-1}\right), s\right), \quad \operatorname{Re} s>1,
\end{aligned}
$$

where $Y=\prod_{X \notin U} X^{A(X)}, F_{X, z}(s)$, for $z \in \mathbf{C}$, is regular and nonvanishing in $s \in D$, and $P_{X, m}(m \geq 0)$ is a polynomial of degree $m$ in the first $h$ variables, with coefficients regular in $s \in D$ and the coefficient at $\log ^{m} \zeta\left(s, \chi_{0}\right)$ constant and equal to $\frac{1}{h^{m} m !}$.

The following theorem allows us to establish the existence of oscillations of an error term of an arithmetic function as defined in the Introduction.

Theorem 3 (11). Let $f(x)$ be a real, piecewise continuous function, defined for $x>0$. Suppose the integral $\int_{0}^{\infty} f(x) x^{-s-1} d x$ is absolutely convergent in a halfplane $\sigma \geq \sigma_{1}$ with $\sigma_{1} \in \mathbf{R}$. Let $F(s)=\int_{0}^{\infty} f(x) x^{-s-1} d x$ in that half-plane and let $\theta \in \mathbf{R}$ be the smallest number such that $F(s)$ can be continued analytically to a function regular in the half-plane $\sigma>\theta$. We assume that $F(s)$ can be analytically continued to a function regular in a larger half-plane $\operatorname{Re} s>\theta-c_{0}\left(c_{0}>0\right)$ with the exclusion of some horizontal cuts starting at its edge. The right ends of the cuts, denoted $\varrho$, contained in the strip $\theta-c_{0} \leq \sigma \leq \theta$, having nonzero imaginary parts and no point of accumulation, are assumed to be singular points of $F(s)$, i.e., $F(s)$ cannot be extended further to a function regular at any of the $\varrho$. In the neighbourhood of radius $\eta_{\varrho}>0$ of a singularity $\varrho$ we assume to have, apart from the cut,

$$
F(s)=\sum_{j=1}^{m_{\varrho}}(s-\varrho)^{w_{\varrho, j}} P_{\varrho, j}(\log (s-\varrho)),
$$

where $m_{\varrho} \geq 1, w_{\varrho, j} \in \mathbf{C}$, and $P_{\varrho, j}$ are polynomials with coefficients regular in the entire $\eta_{\varrho}$-neighbourhood of $\varrho, j=1, \ldots, m_{\varrho}$. Let $\gamma=\min _{\operatorname{Re} \varrho=\theta}|\operatorname{Im} \varrho|$ and $\gamma=\infty$ if there are no singularities on the line $\operatorname{Re} s=\theta$. Then $f(x)$ is subject to oscillations of lower logarithmic frequency greater than or equal to $\frac{\gamma}{\pi}$ and size $x^{\theta-\varepsilon}$.

We also need some other results from [10] and [11] as well as the lemma of J. Kaczorowski and A. Perelli on multiplicities of zeros of functions from the Selberg class.

Lemma 3 (10]). Let $\Omega$ be the interior of $\{s \in \mathbf{C}: \operatorname{Re} s>f(\operatorname{Im} s)\}$ for a real, piecewise continuous function $f$. Suppose $F_{1}, \ldots, F_{k} \in \mathcal{S}$ are regular in $\Omega$. Let 
$G_{1}, \ldots, G_{m}$ be regular in $\Omega$ and nonvanishing in a certain half-plane $\operatorname{Re} s>\sigma_{0} \geq 1$, $\lim _{\sigma \rightarrow+\infty} \arg G_{j}(\sigma)=0, j=1, \ldots, m, P_{1}, \ldots, P_{n}$ polynomials with coefficients regular in $\Omega$, and $\alpha_{i, j}(i=1, \ldots, n, j=1, \ldots, m)$ complex numbers. If the function

$$
Z(s)=\sum_{i=1}^{n}\left(\prod_{j=1}^{m} G_{j}(s)^{\alpha_{i, j}}\right) P_{i}\left(\log F_{1}(s), \ldots, \log F_{k}(s), s\right), \quad \operatorname{Re} s>\sigma_{0},
$$

has a regular continuation in $\Omega$, then

$$
Z(s)=\sum_{i \in I}\left(\prod_{j=1}^{m} G_{j}(s)^{\alpha_{i, j}}\right) P_{i}\left(\log F_{1}(s), \ldots, \log F_{k}(s), s\right), \quad \operatorname{Re} s>\sigma_{0},
$$

where $I=\left\{i \in\{1, \ldots, n\}: \sum_{j=1}^{m} \alpha_{i, j} m\left(\varrho, G_{j}\right) \in \mathbf{Z}, \varrho \in \Omega\right\}$.

Theorem 4 (10]). Let $U_{i} \subset \mathrm{Cl}\left(S_{\Gamma}\right), i=1, \ldots, n, A_{i}: \mathrm{Cl}\left(S_{\Gamma}\right) \backslash U \rightarrow \mathbf{N} \cup\{0\}$, $i=1, \ldots, n$, be such that all $N_{U_{i}, A_{i}}$ are nonempty. Let $M=\max _{\left|U_{i}\right| \neq h}\left|U_{i}\right|$ and

$$
Z(s)=\sum_{i=1}^{n} \alpha_{i} \zeta\left(s, N_{U_{i}, A_{i}}\right), \quad \operatorname{Re} s>1
$$

where $\alpha_{i} \in \mathbf{C}$, with $\alpha_{i}>0$ whenever $\left|U_{i}\right|=M$. If $\max _{\left|U_{i}\right|=M} \sum_{X \notin U_{i}} A_{i}(X)>0$, then $Z(s)$ has infinitely many singularities in the strip $\frac{1}{2} \leq \operatorname{Re} s<1$.

Lemma 4 ([11]). Suppose $\varrho \in \mathbf{C}$ and $\eta>0$. Every function $F$ defined in the neighbourhood $|s-\varrho| \leq \eta$ with the exclusion of the segment $[\varrho-\eta, \varrho]$ by

$$
F(s)=\sum_{j=1}^{m}(s-\varrho)^{w_{j}} P_{j}(\log (s-\varrho))
$$

where $m \geq 0, w_{j} \in \mathbf{C}$, and $P_{j}$ are polynomials with coefficients regular in $|s-\varrho| \leq$ $\eta, j=1, \ldots, m$, can be uniquely represented in the form

$$
F(s)=\sum_{j=1}^{m^{\prime}}(s-\varrho)^{w_{j}^{\prime}} Q_{j}(\log (s-\varrho))
$$

with $m^{\prime}, w_{j}^{\prime}$, and $Q_{j}$ as $m, w_{j}$ and $P_{j}$ above, but $w_{j}^{\prime}\left(j=1, \ldots, m^{\prime}\right)$ pairwise noncongruent $\bmod \mathbf{Z}$ and the coefficients of $Q_{j}\left(j=1, \ldots, m^{\prime}\right)$ not all attaining the value 0 at $\varrho$. Each $w_{j}^{\prime}\left(j=1, \ldots, m^{\prime}\right)$ is congruent $\bmod \mathbf{Z}$ to one of the $w_{j} s . F$ can be analytically continued to a neighbourhood of $\varrho$ if and only if either $m^{\prime}=0$ or $m^{\prime}=1, w_{1}^{\prime}$ is a nonnegative integer and $Q_{1}$ is of degree 0 .

Lemma 5 (Kaczorowski, Perelli 6]). Let $\log F_{1}, \ldots, \log F_{N} \in \log \mathcal{S}$ be linearly independent over $\mathbf{Q}$ and let $\nu(\varrho)=\left(m\left(\varrho, F_{1}\right), \ldots, m\left(\varrho, F_{N}\right)\right)$ for every $\varrho \in \mathbf{C}$. Then there exist infinitely many disjoint $N$-tuples $\left(\varrho_{1}, \ldots, \varrho_{N}\right)$ of nontrivial zeros of $\prod_{j=1}^{N} F_{j}(s)$, with $\operatorname{Re} \varrho_{j} \geq \frac{1}{2}$ for $j=1, \ldots, N$, such that the vectors $\nu\left(\varrho_{1}\right), \ldots, \nu\left(\varrho_{N}\right)$, form a basis of $\mathbf{R}^{N}$. 


\section{Multiplicative independenCE}

In this section we prove Theorem 1 and its corollaries.

Proof of Theorem 1. First, suppose $\chi, \psi \in \widehat{H^{*}(K)}$, and $\chi=\psi \circ \sigma$ for some $\sigma \in$ $\operatorname{Gal}(K / \mathbf{Q})$. Then, obviously

$$
\begin{aligned}
\zeta(s, \chi) & =\sum_{I \in \mathcal{I}\left(\mathcal{O}_{K}\right)} \frac{\chi(I)}{\mathrm{N}(I)^{s}} \\
& =\sum_{I \in \mathcal{I}\left(\mathcal{O}_{K}\right)} \frac{\chi(\sigma(I))}{\mathrm{N}(\sigma(I))^{s}} \\
& =\zeta(s, \psi) .
\end{aligned}
$$

Now suppose $\psi_{1}, \ldots, \psi_{k} \in \widehat{H^{*}(K)}$ are in distinct orbits of the action of $\operatorname{Gal}(K / \mathbf{Q})$ on $\left.\widehat{H^{*}(K}\right)$. We complete the proof of (ii) and (i) by showing that $\log \zeta\left(s, \psi_{1}\right), \ldots$, $\log \zeta\left(s, \psi_{k}\right)$ are linearly independent over $\mathbf{Q}$. Let $\alpha_{1}, \ldots, \alpha_{k} \in \mathbf{Q}$ be such that

$$
\sum_{i=1}^{k} \alpha_{i} \log \zeta\left(s, \psi_{i}\right)=0, \quad \operatorname{Re} s>1,
$$

and let $O$ be any orbit of the aforementioned action. We are going to show that

$$
\sum_{i=1}^{k} \sum_{X \in O} \alpha_{i} \psi_{i}(X)=0 .
$$

Let $\mathfrak{P}_{1}$ be a prime ideal of first degree in a class belonging to $O$, and let $p$ be the prime number lying under $\mathfrak{P}_{1}$. Then we have

$$
p \mathcal{O}_{K}=\mathfrak{P}_{1}^{e} \ldots \mathfrak{P}_{g}^{e}
$$

for some $e, g \in \mathbf{N}, \mathfrak{P}_{1}, \ldots, \mathfrak{P}_{g}$ distinct prime ideals, $\mathrm{N}\left(\mathfrak{P}_{i}\right)=p, i=1, \ldots, g$. We recall that $\operatorname{Gal}(K / \mathbf{Q})$ acts transitively on $\left\{\mathfrak{P}_{1}, \ldots, \mathfrak{P}_{g}\right\}$. The coefficient at $p^{-s}$ in the Dirichlet series of $\log \zeta\left(s, \psi_{i}\right), i=1, \ldots, k$, equals

$$
\begin{aligned}
\sum_{\substack{I \in \mathcal{I}\left(\mathcal{O}_{K}\right) \\
\mathrm{N}(I)=p}} \psi_{i}(I) & =\sum_{j=1}^{g} \psi_{i}\left(\mathfrak{P}_{j}\right) \\
& =\frac{g}{|O|} \sum_{X \in O} \psi_{i}(X),
\end{aligned}
$$

and (4) is clear from (3). Hence, for all $X \in H^{*}(K)$ we have

$$
\sum_{i=1}^{k} \sum_{\sigma \in \operatorname{Gal}(K / \mathbf{Q})} \alpha_{i} \psi_{i}(\sigma(X))=0,
$$

and by linear independence of the characters $\left(\psi_{i} \circ \sigma\right)_{i=1, \ldots, k, \sigma \in \operatorname{Gal}(K / \mathbf{Q})}$ we have $\alpha_{1}=\cdots=\alpha_{k}=0$.

Proof of Corollary 1. By considering decompositions in the form of (5) we see that the automorphisms induced by $\operatorname{Gal}(K / \mathbf{Q})$ on $H^{*}(K)$ are identity and inversion: $X \mapsto X^{-1}$. The result is immediate. 
Proof of Corollary 2, Indeed, let $K$ be a quadratic number field whose ideal class group $H(K)$ is not isomorphic to $C_{2}^{k}, k \in \mathbf{N} \cup\{0\}$. Let $K_{H}$ be the Hilbert class field of $K$. There exists a character $\psi \in \widehat{H(K)}$ such that $\bar{\psi} \neq \psi$. We have

$$
\zeta_{K_{H}}(s)=\prod_{\chi \in \overline{H(K)}} \zeta(s, \chi) .
$$

Hence $\zeta_{K_{H}}(s)$ is divisible by $\zeta(s, \psi) \zeta(s, \bar{\psi})=\zeta(s, \psi)^{2}$, and so has multiple zeros at the points where $\zeta(s, \psi)$ has nontrivial zeros.

\section{Oscillations of THE ERROR TERM OF $G_{1}(x)$}

In this section we prove Theorem 2, For a finite abelian group $G$ and a complexvalued function $f$ defined on $G$ let $\hat{f}$ denote the Fourier transform of $f$, i.e. $\hat{f}$ is defined on the characters group $\widehat{G}$ by

$$
\hat{f}(\chi)=\frac{1}{|G|} \sum_{g \in G} f(g) \bar{\chi}(g) .
$$

Let $\operatorname{char}_{U}$ denote the characteristic function of a subset $U \subset G$. We also use the following equivalence relation in $G: g \sim g^{\prime}$ if $\langle g\rangle=\left\langle g^{\prime}\right\rangle$. We are going to study singularities of functions involving complex powers of the form $(s-\varrho)^{\langle\chi \mid U\rangle m}$ for some $m \in \mathbf{Z}, \chi \in \mathrm{Cl}\left(S_{\Gamma}\right), U \subset \mathrm{Cl}\left(S_{\Gamma}\right), \varrho \in \mathbf{C}$. Rationality of the exponent depends on whether or not the Fourier transform of $\operatorname{char}_{U}$ is rational-valued.

Theorem 5. Let $G$ be a finite abelian group, and let $f$ be a rational-valued function on $G$. Then the image of $\hat{f}$ is contained in $\mathbf{Q}$ if and only if $f$ is constant on the equivalence classes of the relation $\sim$.

Proof. Let $[g]$ denote the equivalence class of $g \in G$ in the relation $\sim$. Let $\mathcal{A}$ be the set of functions $f: G \rightarrow \mathbf{Q}$ such that the image of $\hat{f}$ is contained in $\mathbf{Q}$. $\mathcal{A}$ is closed upon multiplication, because the Fourier transform of $f_{1} f_{2}$, for $f_{1}, f_{2} \in \mathcal{A}$, is a convolution of $\hat{f}_{1}$ and $\hat{f}_{2}$, so its image must be in $\mathbf{Q}$. Clearly $\mathcal{A}$ with the standard addition and multiplication is a commutative algebra with a unit over $\mathbf{Q}$. The assertion states that the characteristic functions of the equivalence classes form a basis of $\mathcal{A}$.

Let $g \in G$. For each $H<\langle g\rangle$ the function $\operatorname{char}_{H}$ belongs to $\mathcal{A}$, as the sum of values of a character over $H$ is either 0 or $|H|$. We have $[g]=\langle g\rangle \backslash \bigcup_{H<\langle g\rangle, H \neq\langle g\rangle} H$, hence

$$
\operatorname{char}_{[g]}=\prod_{\substack{H<\langle g\rangle \\ H \neq\langle g\rangle}}\left(\operatorname{char}_{\langle g\rangle}-\operatorname{char}_{H}\right),
$$

so $\operatorname{char}_{[g]} \in \mathcal{A}$. The functions $\operatorname{char}_{[g]}$ are pairwise orthogonal idempotents that sum up to 1 . Hence

$$
\mathcal{A}=\bigoplus_{[g] \in G / \sim} \operatorname{char}_{[g]} \mathcal{A}
$$

where the sum is taken over distinct equivalence classes. It remains to show that each $f \in \operatorname{char}_{[g]} \mathcal{A}$ is constant on $[g]$. To this end consider $f_{1}=f-f(g) \operatorname{char}_{[g]}$. We have $f_{1} \in \mathcal{A}, f_{1}\left(g^{\prime}\right)=0$ for all $g^{\prime} \in G \backslash \operatorname{char}_{[g]}$, and $f_{1}(g)=0$. Let $n=\operatorname{ord} g$. For 
$k=0, \ldots, n-1$ we have

$$
f_{1}\left(g^{k}\right)=\frac{1}{n} \sum_{i=0}^{n-1} \sum_{j=0}^{n-1} f_{1}\left(g^{i}\right) \mathrm{e}\left(\frac{i j}{n}\right) \mathrm{e}\left(\frac{j k}{n}\right) .
$$

Let $\chi \in \widehat{G}$ be such that $\chi(g)=\mathrm{e}\left(\frac{1}{n}\right)$. Then

$$
\begin{aligned}
f_{1}\left(g^{k}\right) & =\frac{1}{n} \sum_{i=0}^{n-1} \sum_{j=0}^{n-1} f_{1}\left(g^{i}\right) \chi\left(g^{i j}\right) \mathrm{e}\left(\frac{j k}{n}\right) \\
& =\frac{|G|}{n} \sum_{j=0}^{n-1} \hat{f}_{1}\left(\chi^{j}\right) \mathrm{e}\left(\frac{k}{n}\right)^{j} .
\end{aligned}
$$

The polynomial

$$
P(X)=\frac{|G|}{n} \sum_{j=0}^{n-1} \hat{f}_{1}\left(\chi^{j}\right) X^{j}
$$

has rational coefficients and $P\left(e\left(\frac{1}{n}\right)\right)=f_{1}(g)=0$. Hence for each $k=1, \ldots, n$ relatively prime to $n$ we have $f_{1}\left(g^{k}\right)=P\left(e\left(\frac{k}{n}\right)\right)=0$ as $e\left(\frac{k}{n}\right)$ is an algebraic conjugate of $e\left(\frac{1}{n}\right)$. Hence $f_{1}$ is equal to zero and $f$ is constant on $[g]$.

Our main technical result is the following.

Theorem 6. Let $U_{i} \subset \mathrm{Cl}\left(S_{\Gamma}\right), i=1, \ldots, n, A_{i}: \operatorname{Cl}\left(S_{\Gamma}\right) \backslash U \rightarrow \mathbf{N} \cup\{0\}, i=$ $1, \ldots, n$, be such that all $N_{U_{i}, A_{i}}$ are nonempty. Let $M=\max _{\left|U_{i}\right| \neq h}\left|U_{i}\right|$ and

$$
Z(s)=\sum_{i=1}^{n} \alpha_{i} \zeta\left(s, N_{U_{i}, A_{i}}\right), \quad \operatorname{Re} s>1,
$$

where $\alpha_{i} \in \mathbf{C}$, with $\alpha_{i}>0$ whenever $\left|U_{i}\right|=M$. Then at least one of the following is true:

(i) For each $i$ such that $\left|U_{i}\right|=M$ and for each $X, Y \in \mathrm{Cl}\left(S_{\Gamma}\right)$ such that $\langle X\rangle=\langle Y\rangle$ the orbits of $X$ and $Y$ in the action of $\operatorname{Gal}\left(S_{\Gamma}\right)$ have the same number of elements in $U_{i}$.

(ii) The function $Z(s)$ has infinitely many singularities in the strip $\frac{1}{2} \leq \operatorname{Re} s \leq$ 1.

Proof. Let $m(\varrho, \chi)$ denote the multiplicity of a zero of $\zeta(s, \chi)$ at $s=\varrho, \chi \in \widehat{\mathrm{Cl}\left(S_{\Gamma}\right)}$, $\varrho \in \mathbf{C}$. We are going to be brief in the part of the proof similar to the argument in [10]. We can assume $\left|U_{i}\right|<h, i=1, \ldots, n$. Put $Y_{i}=\prod_{X \notin U_{i}} X^{A_{i}(X)}, i=1, \ldots, n$. By Lemma 2 we have

$$
\begin{aligned}
& Z(s)=\frac{1}{h} \sum_{i=1}^{n} \sum_{\chi} \alpha_{i} \chi\left(Y_{i}\right)\left(\prod_{\psi} \zeta(s, \psi)^{\left\langle\chi \bar{\psi} \mid U_{i}\right\rangle}\right)\left(\prod_{X \in U_{i}} F_{X, \chi(X)}(s)\right) \\
& \cdot \prod_{X \notin U_{i}} P_{X, A_{i}(X)}\left(\log \zeta\left(s, \chi_{0}\right), \ldots, \log \zeta\left(s, \chi_{h-1}\right), s\right), \quad \operatorname{Re} s>1 .
\end{aligned}
$$

Suppose (ii) is false, so $Z(s)$ is regular in a region $\Omega$ containing the set

$$
\left\{s \in \mathbf{C}: \operatorname{Re} s \geq \frac{1}{2},|\operatorname{Im} s| \geq T_{1}\right\} \cup\left\{s \in \mathbf{C}: \operatorname{Re} s>1,|\operatorname{Im} s|<T_{1}\right\},
$$


for a $T_{1}>0$. Using Lemma 3 we get

$$
\begin{aligned}
& Z(s)=\frac{1}{h} \sum_{(i, \chi) \in I} \alpha_{i} \chi\left(Y_{i}\right)\left(\prod_{\psi} \zeta(s, \psi)^{\left\langle\chi \bar{\psi} \mid U_{i}\right\rangle}\right)\left(\prod_{X \in U_{i}} F_{X, \chi(X)}(s)\right) \\
& \cdot \prod_{X \notin U_{i}} P_{X, A_{i}(X)}\left(\log \zeta\left(s, \chi_{0}\right), \ldots, \log \zeta\left(s, \chi_{h-1}\right), s\right), \quad \operatorname{Re} s>1,
\end{aligned}
$$

where

$$
I=\left\{(i, \chi) \in\{1, \ldots, n\} \times \widehat{\mathrm{Cl}\left(S_{\Gamma}\right)}: \sum_{\psi} m(\varrho, \psi)\left\langle\chi \bar{\psi} \mid U_{i}\right\rangle \in \mathbf{Z}, \varrho \in \Omega\right\} .
$$

In a neighbourhood of $s=1$ the summands in both expressions for $Z(s)$ in (6) and (17) are of the form $(s-1)^{-\left\langle\chi \mid U_{i}\right\rangle} P_{i, \chi}(\log (s-1), s)$, where $P_{i, \chi}$ is a polynomial in $\log (s-1)$ with regular coefficients. Using the uniqueness part of Lemma 4 in the neighbourhood of $s=1$ we can compare the sums of terms on the right-hand sides of (6) and (77) where the factors $(s-1)^{-\left\langle\chi \mid U_{i}\right\rangle} \log ^{k}(s-1)$ occur with $\left\langle\chi \mid U_{i}\right\rangle \equiv \frac{M}{h}$ $(\bmod \mathbf{Z})$ and $k=0$. Note that $\left\langle\chi \mid U_{i}\right\rangle=\frac{M}{h}$ is only possible if $\left|U_{i}\right|=M$ and $\left\langle U_{i}\right\rangle \subset \operatorname{ker} \chi$, and then we have $\sum A_{i}(X)=0$ by Theorem 4 . We obtain

$$
\begin{aligned}
& \sum_{\substack{(i, \chi) \notin I \\
\left\langle\chi \mid U_{i}\right\rangle=\frac{M}{h}}} \alpha_{i}\left(\prod_{\psi} \zeta(s, \psi)^{\left\langle\bar{\psi} \mid U_{i}\right\rangle}\right)\left(\prod_{X \in U_{i}} F_{X, 1}(s)\right) \\
&+\sum_{\substack{(i, \chi) \notin I \\
\left\langle\chi \mid U_{i}\right\rangle=\frac{M}{h}-1}}(s-1)^{1-\frac{M}{h}} P_{i, \chi}(0, s)=0,
\end{aligned}
$$

in a neighbourhood of $s=1$. Thus

$$
\lim _{\sigma \rightarrow 1^{+}} \sum_{\substack{(i, \chi) \notin I \\\left\langle\chi \mid U_{i}\right\rangle=\frac{M}{h}}} \alpha_{i}(\sigma-1)^{\frac{M}{h}}\left(\prod_{\psi} \zeta(\sigma, \psi)^{\left\langle\bar{\psi} \mid U_{i}\right\rangle}\right)\left(\prod_{X \in U_{i}} F_{X, 1}(\sigma)\right)=0 .
$$

However, each summand above has a positive limit as $\sigma \rightarrow 1^{+}$, so the sum must be empty. Let $i, \chi$ be such that $\left|U_{i}\right|=M$ and $\left\langle U_{i}\right\rangle \subset \operatorname{ker} \chi$. We must have

$$
\sum_{\psi} m(\varrho, \psi)\left\langle\bar{\psi} \mid U_{i}\right\rangle \in \mathbf{Z}, \quad \varrho \in \Omega
$$

Suppose $\log \zeta\left(s, \chi_{0}\right), \ldots, \log \zeta\left(s, \chi_{l}\right)$ is a maximal linearly-independent subset of $\log \zeta\left(s, \chi_{0}\right), \ldots, \log \zeta\left(s, \chi_{h-1}\right)$ and let $O_{j}, j=0, \ldots, l$, be the orbit of $\chi_{j}$ in the action of $\operatorname{Gal}\left(S_{\Gamma}\right)$ on $\mathrm{Cl}\left(S_{\Gamma}\right)$. Then we have

$$
\sum_{j=0}^{l} m\left(\varrho, \chi_{j}\right) \sum_{\psi \in O_{j}}\left\langle\bar{\psi} \mid U_{i}\right\rangle \in \mathbf{Z}, \quad \varrho \in \Omega,
$$

by Theorem 1, part (ii), and

$$
\sum_{\psi \in O_{j}}\left\langle\bar{\psi} \mid U_{i}\right\rangle \in \mathbf{Q}, \quad j=0, \ldots, l,
$$


by Lemma 5 . Hence

$$
\sum_{\sigma \in \operatorname{Gal}\left(S_{\Gamma}\right)}\left\langle\bar{\psi} \circ \sigma \mid U_{i}\right\rangle=\sum_{\sigma \in \operatorname{Gal}\left(S_{\Gamma}\right)}\left\langle\bar{\psi} \mid \sigma\left(U_{i}\right)\right\rangle \in \mathbf{Q}, \quad \psi \in \widehat{\operatorname{Cl}\left(S_{\Gamma}\right)} .
$$

Thus the function $\sum_{\sigma \in \operatorname{Gal}\left(S_{\Gamma}\right)} \operatorname{char}_{\sigma\left(U_{i}\right)}$ must be constant on equivalence classes of the relation $\sim$ by Theorem 5 , and the assertion (i) follows.

To prove our main result, Theorem 2, we apply Theorem 6 to

$$
Z(s)=\zeta\left(s, G_{1}\right)=\sum_{i=1}^{m} \alpha_{i} \zeta\left(s, N_{U_{i}, A_{i}}\right)
$$

for appropriate $\alpha_{i}, U_{i}, A_{i}$; cf. Lemma 1. This implies the existence of singularities of $\zeta\left(s, G_{1}\right)$ and, in turn, of

$$
\frac{1}{s} \zeta\left(s, G_{1}\right)=\int_{0}^{\infty} G_{1}(x) x^{-s-1} d x
$$

and

$$
\int_{0}^{\infty}\left(G_{1}(x)-M(x)\right) x^{-s-1} d x
$$

The assertion now follows from Theorem 3, cf. also the analogous argument in [10].

Proof of Corollary 3, Let $U$ and $X \in U$ be as assumed. First we show that for each $\sigma \in \operatorname{Gal}\left(S_{\Gamma}\right)$ there exists at most one $k \in \mathbf{N}, k<\operatorname{ord} X$, relatively prime to ord $X$, such that $\sigma\left(X^{k}\right) \in U$. Indeed, if $\sigma\left(X^{k}\right) \in U$ and $\sigma\left(X^{k^{\prime}}\right) \in U, k^{\prime} \neq k$, then let $a \in \mathbf{N}, a<\operatorname{ord} X$ be such that $k a \equiv-k^{\prime}(\bmod \operatorname{ord} X)$. We have $a \leq \operatorname{ord} X-2$, so considering the block $\sigma\left(X^{k}\right)^{a} \sigma\left(X^{k^{\prime}}\right)$ we see that

$$
\frac{a}{\operatorname{ord} X}+\frac{1}{\operatorname{ord} X}<1
$$

a contradiction with half-factoriality of $U$.

Since $\varphi(\operatorname{ord} X)>\left|\operatorname{Gal}\left(S_{\Gamma}\right)\right|$ there exists a $k$ as above such that $\sigma\left(X^{k}\right) \notin U$, $\sigma \in \operatorname{Gal}\left(S_{\Gamma}\right)$. We have $\left\langle X^{k}\right\rangle=\langle X\rangle$ and the orbit of $X^{k}$ in the action of $\operatorname{Gal}\left(S_{\Gamma}\right)$ has no common elements with $U$ while the orbit of $X$ contains $X \in U$. The assertion follows from Theorem 2 ,

Proof of Corollary 4, In this case we have $\left|\operatorname{Gal}\left(S_{\Gamma}\right)\right| \leq|\operatorname{Gal}(K / \mathbf{Q})| \leq 3$. The equation $\varphi(\operatorname{ord} X) \leq 3$ is equivalent to $\varphi(\operatorname{ord} X) \leq 2$ and has no other solutions but those listed in the assertion.

\section{REFERENCES}

1. L. Carlitz, A characterization of algebraic number fields with class number two, Proc. Amer. Math. Soc. 11 (1960), 391-392. MR0111741 (22:2603)

2. W. Gao and A. Geroldinger, Half-factorial domains and half-factorial subsets of abelian groups, Houston J. Math. 24 (1998), no. 4, 593-611. MR.1686626 (2000e:13027)

3. A. Geroldinger and F. Halter-Koch, Congruence monoids, Acta Arith. 112 (2004), no. 3, 263-296. MR2046184 (2005b:11175)

4. F. Halter-Koch, Halbgruppen mit Divisorentheorie, Exposition. Math. 8 (1990), no. 1, 27-66. MR 1042201 (91c:20091)

5. J. Kaczorowski, Some remarks on factorization in algebraic number fields, Acta Arith. 43 (1983), no. 1, 53-68. MR0730848 (85j:11141)

6. J. Kaczorowski and A. Perelli, Functional independence of the singularities of a class of Dirichlet series, Amer. J. Math. 120 (1998), no. 2, 289-303. MR1613634 (99b:11102) 
7. - The Selberg class: a survey, Number theory in progress, Vol. 2 (Zakopane-Kościelisko, 1997), de Gruyter, Berlin, 1999, pp. 953-992. MR1689554 (2001g:11141)

8. W. Narkiewicz, Finite abelian groups and factorization problems, Colloq. Math. 42 (1979), 319-330. MR0567570 (81i:12006)

9. _ Elementary and analytic theory of algebraic numbers, second ed., Springer-Verlag, Berlin, 1990. MR1055830 (91h:11107)

10. M. Radziejewski, On the distribution of algebraic numbers with prescribed factorization properties, Acta Arith. 116 (2005), no. 2, 153-171. MR2110393 (2005k:11204)

11. Oscillations of error terms associated with certain arithmetical functions, Monatsh. Math. 144 (2005), no. 2, 113-130. MR2123959 (2005k:11205)

12. M. Radziejewski and W. A. Schmid, On the asymptotic behavior of some counting functions, Colloq. Math. 102 (2005), no. 2, 181-195. MR2152660 (2006d:11114)

13. L. Skula, On c-semigroups, Acta Arith. 31 (1976), no. 3, 247-257. MR0444817 (56:3164)

14. J. Śliwa, Factorizations of distinct lengths in algebraic number fields, Acta Arith. 31 (1976), no. 4, 399-417. MR 0429830 (55:2840)

15. - Remarks on factorizations in algebraic number fields, Colloq. Math. 46 (1982), no. 1, 123-130. MR0672372 (84c:12004)

16. A. Zaks, Half factorial domains, Bull. Amer. Math. Soc. 82 (1976), no. 5, 721-723. MR0407001 (53:10785a)

Faculty of Mathematics and Computer Science, Adam Mickiewicz University, UmulTOWSKa 87, 61-614 Poznań, POLAND

E-mail address: maciejr@amu.edu.pl 